\title{
Spatial linear global instability analysis of the HIFiRE-5 elliptic cone model flow
}

\author{
P. Paredes ${ }^{*}$ and V. Theofilis ${ }^{\dagger}$ \\ School of Aeronautics, Universidad Politécnica de Madrid, E-28040 Madrid, Spain
}

\begin{abstract}
The linear instability of the three-dimensional boundary-layer over the HIFiRE-5 flight test geometry, i.e. a rounded-tip 2:1 elliptic cone, at Mach 7, has been analyzed through spatial BiGlobal analysis, in a effort to understand transition and accurately predict local heat loads on next-generation flight vehicles. The results at an intermediate axial section of the cone, $R e_{x} \approx 8 \times 10^{5}$, show three different families of spatially amplified linear global modes, the attachment-line and cross-flow modes known from earlier analyses, and a new global mode, peaking in the vicinity of the minor axis of the cone, termed "center-line mode". We discover that a sequence of symmetric and anti-symmetric centerline modes exist and, for the basic flow at hand, are maximally amplified around $F^{*}=130 \mathrm{kHz}$. The wavenumbers and spatial distribution of amplitude functions of the centerline modes are documented.
\end{abstract}

\section{Nomenclature}

Abbreviations

$P S E \quad$ Parabolized Stability Equations

$F D-q \quad$ Stable high-order Finite Differences

$P N S \quad$ Parabolic Navier-Stokes equations

GEVP Generalized EigenValue Problem

Latin Symbols

q Instantaneous flow vector

$\overline{\mathbf{q}} \quad$ Base flow vector

$\hat{\mathbf{q}} \quad$ Vector of amplitude functions

Greek Symbols

$\omega \quad$ Circular frequency

$\mathcal{R}(\alpha) \quad$ Wavenumber

$\mathcal{I}(\alpha) \quad$ Spatial growth/damping rate

$\Theta \quad$ Phase function

Superscript

$\dagger \quad$ Complex conjugate

*Student Member AIAA. Correspondence to: pedro.paredes@upm.es

${ }^{\dagger}$ Research Professor, Associate Fellow AIAA 


\section{Introduction}

Prediction of laminar-turbulent flow transition and the associated heat-transfer in high-speed flows, as well as control of both phenomena, is key to optimizing performance of next-generation aerospace vehicles. The elliptic cone is a frequently used model to understand transition over components of such vehicles. Evidence has been accumulated regarding laminar-turbulent transition scenarios on elliptic cones at aspect ratios 2:1 and 4:1, exposed at zero angle of attack to oncoming flows for Mach numbers $(M a)$ between 4 and 8 in different experimental facilities, ${ }^{1,2,3,4}$ while recently large-scale computations of the same phenomenon have appeared in the literature. ${ }^{5}$ All these studies reported the formation of large structures near the minor axis center-line of the cone; these structures were first experimentally found by Schmisseur et al. ${ }^{1,2}$ to be most receptive to amplification of perturbations in a 4:1 elliptic cone at $M a=4$. Simultaneously, Poggie \& Kimmel $^{3}$ reported evidence of the classical cross-flow and second Mack mode ${ }^{6}$ instabilities in a 2:1 elliptic cone at $M a=8$; the transition front was asymmetric, with early transition near the top centerline and delayed transition near the leading edge. Images taken by Huntley \& Smits ${ }^{4}$ of the early stages of transition, on a sharp-nosed 4:1 elliptic cone at same $M a=8$, confirm that transition begins with the emergence of small-scale structures near the centerline axis of the cone, rather than in the outboard cross-flow region.

In the last two decades, stability analyses of boundary layers on sharp-nosed cones with elliptical cross sections have been performed, using linear stability theory and cross flow correlations. These calculations relied on mean flow solutions recovered using the Parabolized Navier-Stokes equations. In 90's of last century, research into three-dimensional boundary-layers over elliptic cross-section cones was undertaken by Lyttle \& Reed, ${ }^{7}$ who presented solutions of the PNS equations for adiabatic wall elliptic cones of eccentricities of 2:1, 3:1 and 4:1 at $M a=4$, applying Reynolds number correlations based on the parameter $R_{c f(R \& H)}=H L R e_{c f}$, where $R e_{c f}$ is the traditional cross-flow Reynolds number and the factors $H$ and $L$ are introduced to account for compressibility and cooling effects of the wall (see Reed \& Haynes ${ }^{8}$ ), for stability analysis. The parameter $R e_{c f(R \& H)}$ for these configurations peaked near the top center-line, outside the region of validity of the above correlation. Boundary layer velocity profiles near the top centerline were inflectional and unstable. Kimmel et $a l .{ }^{9}$ used an extended version of the the UPS PNS code, ${ }^{10,11}$ enabling the study of cool-wall cases, for computing the base flow around cones with eccentricities of 1.5:1, 2:1 and 4:1 at $M a=7.95$. The $e^{\text {Malik }}$ $\operatorname{code}^{12}$ was used to calculate boundary layer stability, demonstrating that all the three cases showed crossflow instability, with the 4:1 configuration attaining the highest $N$-factors. Later experimental studies by the same authors ${ }^{13}$ delivered results in reasonable agreement with linear stability calculations and were suggestive of a traveling cross-flow instability mode. The flow in the vicinity of the top centerline was found to be highly unstable and for this region both the experiments and the computations showed an unstable frequency band that coincided with the characteristic second Mack mode ${ }^{6}$ frequency.

Recently, Gosse \& Kimmel $^{14}$ compared the mean flow and transition correlating parameters of a 2:1 elliptic cone at a free-stream Mach number of 7.95 calculated using both a full Navier-Stokes CFD solver and a PNS code, resulting in good agreement. From then on, efforts have concentrated on the HIFiRE5 geometry, also studied in this work. Details of the HIFiRE-5 configuration are discussed in Kimmel et al..$^{15}$ The works of Choudhari et al. ${ }^{16}$ and $\mathrm{Li}$ et al. ${ }^{17}$ present a thorough instability analysis of this configuration using local and non-local theories, respectively based on the solution of the one-dimensional eigenvalue problem or the Parabolized Stability Equations. These approaches base the transition prediction on the $N$-factor over streamlines or grid lines of the cone, mainly the top center-line or the attachment-line. Furthermore, in $^{16}$ also a two-dimensional stability theory is used, focusing on the flow features near the top center-line and the leading-edge, without accounting for surface curvature effects. The two-dimensional global linear eigenvalue problem (called here BiGlobal analysis ${ }^{18}$ ) results show unstable modes in both cases.

Recent large-scale computations by Bartkowicz et al. ${ }^{5}$ confirm the co-existence of all these scenarios and attempt a first classification of their significance at different Reynolds number $(R e)$ range: while the center-line structures lead flow to transition at lower $R e$ values, cross-flow instability near the elliptic cone leading-edge becomes competitive at higher Reynolds numbers. The origin and role of the large center-line structures in the laminar-turbulent transition process on the elliptic cone is presently unclear. Mapping of the parameter space with respect to critical conditions and study of nonlinear interactions of different modal scenarios potentially leading flow to transition are issues hardly to be addressed by large-scale computations; spatial BiGlobal linear analysis and three-dimensional Parabolized Stability Equations (PSE-3D) are called for accomplish these tasks. 
In this work, the hypersonic flow over an elliptic cone is analyzed using spatial BiGlobal analysis for the first time, including surface curvature effects. The BiGlobal analysis theory assumes that the base flow is independent of only one spatial coordinate, $x$, corresponding in this case with the direction of the free-stream flow, since the angle of attack is zero. The in-house developed stability code has been recently validated against DNS results analyzing instability of the wake behind an isolated roughness element in supersonic flow. ${ }^{19}$ In the latter work, the first three-dimensional Parabolized Stability Equations (PSE-3D) analysis for high-speed flows is performed, showing excellent agreement when compared with DNS results in the linear regime.

The spatial BiGlobal equations are formulated for compressible flows in Section II. The numerical solution procedure of the spatial BiGlobal equations and spatial discretization are presented in Section III. Clarifications about the analyzed mean flow and performed nondimensionalization are exposed in Section IV. Section V shows spectra and eigenmode shape functions for a wide range of frequencies at an intermediate streamwise position of the cone. Three different families of unstable eigenmodes are identified: the first two, the attachment-line and the cross-flow modes are known from earlier analyses ${ }^{16}$ while a new linear eigenmode is identified, termed the center-line mode, which is accessible only to the present type of global eigenvalue problem solutions. Attention is thus focused on documenting the characteristics of the centerline mode, including wavenumber and amplitude function distribution of a sequence of symmetric and anti-symmetric centerline modes first discovered herein. Summary and concluding remarks are offered in Section VI.

\section{Instability analyses}

The analysis of flow stability is based on the compressible equations of motion, written in dimensionless form as

$$
\begin{aligned}
\frac{\partial \rho}{\partial t}+\nabla \cdot(\rho \mathbf{V}) & =0 \\
\rho\left[\frac{\partial \mathbf{V}}{\partial t}+(\mathbf{V} \cdot \nabla) \mathbf{V}\right] & =-\nabla p+\frac{1}{R e}\left\{\nabla[\lambda(\nabla \cdot \mathbf{V})]+\nabla \cdot\left[\mu\left((\nabla \mathbf{V})+(\nabla \mathbf{V})^{\mathbf{T}}\right)\right]\right\}, \\
\rho\left[\frac{\partial T}{\partial t}+(\mathbf{V} \cdot \nabla) \mathbf{T}\right] & =\frac{1}{\operatorname{RePr}} \nabla \cdot(\kappa \nabla T)+(\gamma-1) M^{2}\left[\frac{\partial p}{\partial t}+(\mathbf{V} \cdot \nabla) p\right] \\
& +\frac{(\gamma-1) M^{2}}{\operatorname{Re}}\left\{\lambda(\nabla \cdot \mathbf{V})^{2}+\frac{\mu}{\mathbf{2}}\left[(\nabla \mathbf{V})+(\nabla \mathbf{V})^{\mathbf{T}}\right]^{2}\right\}
\end{aligned}
$$

where $\mathbf{V}$ is the velocity vector, $\rho$ the density, $p$ the pressure, $T$ the temperature, $M a$ the Mach number, $R e$ the Reynolds number $\operatorname{Pr}$ the Prandtl number, $\gamma$ the specific heat coefficient, $\kappa$ the thermal conductivity, $\mu$ the first coefficient of viscosity and $\lambda$ the second coefficient of viscosity. The equation of state is given by the perfect gas relation $p=\rho T /\left(\gamma M^{2}\right)$. Note that using the Stoke's law $\lambda=-2 / 3 \mu$. The Sutherland's law is used for the viscosity coefficient

$$
\mu=(T)^{3 / 2} \frac{1+S}{T+S}
$$

with $\mu_{r}^{*}=1.716 \times 10^{-5} \mathrm{~N} \mathrm{~s} / \mathrm{m}^{2}, T_{r}^{*}=273.15 \mathrm{~K}$ and $S=110.4 \mathrm{~K} / T_{r}^{*}$ for air in standard conditions.

The development in time and space of small-amplitude perturbations superposed upon a given flow can be described by the linearized Navier-Stokes equations. Linearization of the equations of motion is performed around a laminar steady flow, denoted as base flows, $\overline{\mathbf{q}}=(\bar{\rho}, \bar{u}, \bar{v}, \bar{w}, \bar{T})^{T}$.

In using the term small-amplitude perturbations, solutions to the initial-value-problem

$$
\mathcal{B}(R e, M a, \overline{\mathbf{q}}) \frac{d \hat{\mathbf{q}}}{d t}=\mathcal{A}(R e, M a, \overline{\mathbf{q}}) \hat{\mathbf{q}},
$$

are denoted, where $\hat{\mathbf{q}}(x, y, z, t)=(\hat{\rho}, \hat{u}, \hat{v}, \hat{w}, \hat{T})^{T}$ is the vector comprising the amplitude functions of linear density, velocity components and temperature perturbations; see ${ }^{18}$ for more details. 


\section{A. Coordinate transformation}

The code is developed for a general three-dimensional coordinate transformation of the form

$$
\xi=\xi(x), \quad \eta=\eta(x, y, z), \quad \zeta=\zeta(x, y, z),
$$

which is used to transform the governing equations into the $(\xi, \eta, \zeta)$ system. This transformation restricts the $(\eta, \zeta)$ solution surfaces to be in a plane normal to the $x$ axis. Using the chain rule of partial differentiation, the first order partial derivatives become

$$
\frac{\partial}{\partial x}=\xi_{x} \frac{\partial}{\partial \xi}+\eta_{x} \frac{\partial}{\partial \eta}+\zeta_{x} \frac{\partial}{\partial \zeta}, \quad \frac{\partial}{\partial y}=\eta_{y} \frac{\partial}{\partial \eta}+\zeta_{y} \frac{\partial}{\partial \zeta}, \quad \frac{\partial}{\partial z}=\eta_{z} \frac{\partial}{\partial \eta}+\zeta_{z} \frac{\partial}{\partial \zeta},
$$

Note that $\xi_{y}=\xi_{z}=0$. The metrics $\left(\xi_{x}, \eta_{x}, \eta_{y}, \eta_{z}, \zeta_{x}, \zeta_{y}, \zeta_{z}\right)$ appearing in these equations are determined using

$$
\left(\begin{array}{ccc}
\xi_{x} & 0 & 0 \\
\eta_{x} & \eta_{y} & \eta_{z} \\
\zeta_{x} & \zeta_{y} & \zeta_{z}
\end{array}\right)=\left(\begin{array}{ccc}
x_{\xi} & 0 & 0 \\
y_{\xi} & y_{\eta} & y_{\zeta} \\
z_{\xi} & z_{\eta} & z_{\zeta}
\end{array}\right)^{-1}=J\left(\begin{array}{ccc}
y_{\eta} z_{\zeta}-y_{\zeta} z_{\eta} & 0 & 0 \\
-\left(y_{\xi} z_{\zeta}-y_{\zeta} z_{\xi}\right) & x_{\xi} z_{\zeta} & -x_{\xi} y_{\zeta} \\
y_{\xi} z_{\eta}-y_{\eta} z_{\xi} & x_{\xi} z_{\eta} & x_{\xi} y_{\eta}
\end{array}\right)
$$

where $J$ is the Jacobian of the transformation, which can be evaluated in the following manner

$$
J=\frac{\partial(\xi, \eta, \zeta)}{\partial(x, y, z)}=1 / J^{-1}=1 / \frac{\partial(x, y, z)}{\partial(\xi, \eta, \zeta)}=1 /\left|\begin{array}{ccc}
x_{\xi} & 0 & 0 \\
y_{\xi} & y_{\eta} & y_{\zeta} \\
z_{\xi} & z_{\eta} & z_{\zeta}
\end{array}\right|=1 /\left[x_{\xi}\left(y_{\eta} z_{\zeta}-y_{\zeta} z_{\eta}\right)\right] .
$$

In order to express second order derivatives in terms of computational coordinate derivatives, the next equivalence, written using Einstein notation, is used:

$$
\frac{\partial f}{\partial x_{i}}=\xi_{x_{i}}^{j} \frac{\partial f}{\partial \xi^{j}}=J \frac{\partial}{\partial \xi^{j}}\left(\frac{\xi_{x_{i}}^{j} f}{J}\right)-J f\left[\frac{\partial}{\partial \xi^{j}}\left(\frac{\xi_{x_{i}}^{j}}{J}\right)\right]
$$

where $f=f(x, y, z)$ is an arbitrary scalar function, $i=1,2,3, j=1,2,3,\left(x_{1}, x_{2}, x_{3}\right)=(x, y, z)$ and $\left(\xi^{1}, \xi^{2}, \xi^{3}\right)=(\xi, \eta, \zeta)$. The term in square brackets is equal to zero. This can be verified by substituting the metrics given by Eq. (8) into this term. Then, using the above expression (10), the second order derivative with respect to the physical coordinates $x_{i}$ and $x_{j}$ of an arbitrary scalar function $f$ is expressed as follow

$$
\frac{\partial^{2} f}{\partial x_{i} \partial x_{j}}=\xi_{x_{i}}^{k} \frac{\partial}{\partial \xi^{k}}\left(\xi_{x_{j}}^{l} \frac{\partial f}{\partial \xi^{l}}\right)=J \frac{\partial}{\partial \xi^{k}}\left(\frac{\xi_{x_{i}}^{k} \xi_{x_{j}}^{l} \frac{\partial f}{\partial \xi^{l}}}{J}\right)=\xi_{x_{i}}^{k} \xi_{x_{j}}^{l} \frac{\partial^{2} f}{\partial \xi^{k} \partial \xi^{l}}+J \frac{\partial}{\partial \xi^{k}}\left(\frac{\xi_{x_{i}}^{k} \xi_{x_{j}}^{l}}{J}\right) \frac{\partial f}{\partial \xi^{l}} .
$$

\section{B. Spatial BiGlobal analysis}

Spatial BiGlobal analysis is the analog of classic spatial linear theory in a local framework, ${ }^{20}$ in case two inhomogeneous spatial directions are resolved simultaneously on a plane, while the third direction is considered locally homogeneous. In the elliptic cone geometry both the plane of amplitude functions and the homogeneous spatial direction are defined on a transformed coordinate system as follows. The transformed coordinate system $(\xi, \eta, \zeta)$ is taken such that

$$
L_{\xi} \gg L_{\eta}, L_{\zeta}, \quad \partial() / \partial \xi \ll \partial() / \partial \eta, \partial() / \partial \zeta,
$$

where $L_{\xi}, L_{\eta}$ and $L_{\zeta}$ are the characteristic lengths on the streamwise and normal to it spatial directions respectively. In order to proceed, the base flow is assumed to be locally independent of one spatial coordinate $\xi$ (but depending on the other two spatial directions, $\eta$ and $\zeta$, in a coupled inhomogeneous manner). Flow quantities are then decomposed according to

$$
\mathbf{q}(\xi, \eta, \zeta, t)=\overline{\mathbf{q}}(\eta, \zeta)+\varepsilon \tilde{\mathbf{q}}(\xi, \eta, \zeta, t), \quad \varepsilon \ll 1,
$$

where $\varepsilon \tilde{\mathbf{q}}$ represents the unsteady three-dimensional infinitesimal perturbations, being inhomogeneous in $\eta$ and $\zeta$ and periodic in $\xi$. Thus, one may write

$$
\tilde{\mathbf{q}}(\xi, \eta, \zeta, t)=\hat{\mathbf{q}}(\eta, \zeta) \Theta(\xi, t)+\text { c.c. }
$$


with $\hat{\mathbf{q}}$ representing the vector of two-dimensional complex amplitude functions and

$$
\Theta=\exp [\mathrm{i}(\alpha \xi-\omega t)]
$$

a complex phase function.

The linear disturbance equations of spatial BiGlobal stability analysis are obtained at $\mathcal{O}(\varepsilon)$ by substituting (13) into the governing equations, subtracting out the $\mathcal{O}(1)$ base flow terms and neglecting terms at $\mathcal{O}\left(\varepsilon^{2}\right)$. In the present spatial framework, $\omega$ is taken to be a real circular frequency parameter, while the complex eigenvalue $\alpha$, and the associated eigenvectors $\hat{\mathbf{q}}$ are sought. The real part of the eigenvalue $\alpha_{r}$ is related with the wavenumber of the eigenmode along the homogeneous spatial direction $\xi, \alpha_{r}=2 \pi / L_{\xi}$, while the imaginary part is its growth/damping rate; a negative value of $\alpha_{i}$ indicates exponential growth of $\tilde{\mathbf{q}}$ in space, while $\alpha_{i}>0$ denotes decay of $\tilde{\mathbf{q}}$ in space.

The resulting two-dimensional partial derivative Generalized Eigenvalue Problem (GEVP) is nonlinear on eigenvalue $\alpha$, but it is converted into a linear eigenvalue problem, which is larger in size by a factor equal to the degree of nonlinearity $\left(\mathrm{see}^{21}\right.$ ), using the companion matrix method, ${ }^{22}$ in which an auxiliary vector is defined, $\hat{\mathbf{q}}^{+}=[\hat{\rho}, \hat{u}, \hat{v}, \hat{w}, \hat{T}, \alpha \hat{u}, \alpha \hat{v}, \alpha \hat{w}, \alpha \hat{T}]^{T}$, and the resulting GEVP is

$$
\begin{gathered}
\left(\begin{array}{ccccccccc}
\mathcal{A}_{11} & \mathcal{A}_{12} & \mathcal{A}_{13} & \mathcal{A}_{14} & 0 & 0 & 0 & 0 & 0 \\
\mathcal{A}_{21} & \mathcal{A}_{22} & \mathcal{A}_{23} & \mathcal{A}_{24} & \mathcal{A}_{25} & 0 & 0 & 0 & 0 \\
\mathcal{A}_{31} & \mathcal{A}_{32} & \mathcal{A}_{33} & \mathcal{A}_{34} & \mathcal{A}_{35} & 0 & 0 & 0 & 0 \\
\mathcal{A}_{41} & \mathcal{A}_{42} & \mathcal{A}_{43} & \mathcal{A}_{44} & \mathcal{A}_{45} & 0 & 0 & 0 & 0 \\
\mathcal{A}_{51} & \mathcal{A}_{52} & \mathcal{A}_{53} & \mathcal{A}_{54} & \mathcal{A}_{55} & 0 & 0 & 0 & 0 \\
0 & 0 & 0 & 0 & 0 & \mathcal{I} & 0 & 0 & 0 \\
0 & 0 & 0 & 0 & 0 & 0 & \mathcal{I} & 0 & 0 \\
0 & 0 & 0 & 0 & 0 & 0 & 0 & \mathcal{I} & 0 \\
0 & 0 & 0 & 0 & 0 & 0 & 0 & 0 & \mathcal{I}
\end{array}\right)\left(\begin{array}{c}
\hat{\rho} \\
\hat{u} \\
\hat{v} \\
\hat{w} \\
\hat{T} \\
\alpha \hat{u} \\
\alpha \hat{v} \\
\alpha \hat{w} \\
\alpha \hat{T}
\end{array}\right)= \\
\alpha\left(\begin{array}{ccccccccc}
\mathcal{B}_{11} & \mathcal{B}_{12} & 0 & 0 & 0 & 0 & 0 & 0 & 0 \\
\mathcal{B}_{21} & \mathcal{B}_{22} & \mathcal{B}_{23} & \mathcal{B}_{24} & \mathcal{B}_{25} & \mathcal{B}_{26} & 0 & 0 & 0 \\
0 & \mathcal{B}_{32} & \mathcal{B}_{33} & 0 & \mathcal{B}_{35} & 0 & \mathcal{B}_{37} & 0 & 0 \\
0 & \mathcal{B}_{42} & 0 & \mathcal{B}_{44} & \mathcal{B}_{45} & 0 & 0 & \mathcal{B}_{48} & 0 \\
\mathcal{B}_{51} & \mathcal{B}_{52} & \mathcal{B}_{53} & \mathcal{B}_{54} & \mathcal{B}_{55} & 0 & 0 & 0 & \mathcal{B}_{59} \\
0 & \mathcal{I} & 0 & 0 & 0 & 0 & 0 & 0 & 0 \\
0 & 0 & \mathcal{I} & 0 & 0 & 0 & 0 & 0 & 0 \\
0 & 0 & 0 & \mathcal{I} & 0 & 0 & 0 & 0 & 0 \\
0 & 0 & 0 & 0 & \mathcal{I} & 0 & 0 & 0 & 0
\end{array}\right)\left(\begin{array}{c}
\hat{\rho} \\
\hat{u} \\
\hat{v} \\
\hat{w} \\
\hat{T} \\
\alpha \hat{u} \\
\alpha \hat{v} \\
\alpha \hat{w} \\
\alpha \hat{T}
\end{array}\right)
\end{gathered}
$$

The entries of $\mathcal{A}_{2 D}$ and $\mathcal{B}_{2 D}$ are found in. ${ }^{23}$

\section{Numerical considerations}

\section{A. Elliptic cone transformation}

The computational domain coordinate system $(\xi, \eta, \zeta)$ is transformed into the desired physical coordinate system $(x, y, z)$ using a modified confocal elliptic transformation, written as follows

$$
x=\xi, \quad y=c \xi \sinh \left(\eta_{0}+s p(\zeta) \eta\right) \sin \zeta, \quad z=c \xi \cosh \left(\eta_{0}+s p(\zeta) \eta\right) \cos \zeta,
$$

where $c$ sets the half angle of the cone minor-axis, $c=\operatorname{atan} \alpha / \sinh \eta_{0}, s p(\zeta)$ sets the location where the calculation domain is truncated and $\eta_{0}$ is a parameter controlling the Aspect Ratio $(A R)$ of the cone, $\eta_{0}=\operatorname{atanh}(1 / A R)$. 


\section{B. Boundary conditions}

The elliptic eigenvalue problem (16) must be complemented with adequate boundary conditions for the disturbance variables. Dealing firstly with the azimuthal direction, $\zeta$, the symmetries of the problem are exploited in order to reduce the computational requirements. Depending on the case, either symmetric or antisymmetric boundary for each flow component, the corresponding homogeneous Dirichlet or Neumann boundary conditions are imposed at $\zeta=0$ and $\zeta=\pi / 2$. The combination of these conditions gives rise to four cases, but it is reduced to two due to the linearity of the modes and the clear independence of the structure of the shape functions either if it is located near $\zeta=0$ or $\zeta=\pi / 2$ as will be discussed in Section V. For the wall-normal direction, the perturbations are forced to decay through the imposition of a sponge region outside the shock layer in the free-stream region, setting homogeneous Dirichlet boundary conditions at $\eta=1$. The main objective of this sponge region is to avoid spurious reflections. This is achieved by artificially decreasing the local Reynolds number using a smoothing function. At the wall, $\eta=0$, no-slip conditions are imposed by setting homogeneous Dirichlet boundary conditions and the same condition is set for temperature amplitude function. No boundary condition needs to be imposed for density amplitude function at the wall, since the linearized continuity equation is satisfied at $\eta=0$.

\section{Large matrix inversion and spatial discretization}

The inversion of the matrices discretizing the two-dimensional PDE of the spatial BiGlobal analysis GEVP, which leading dimension is $\mathcal{O}\left(10^{4}-10^{5}\right)$, is performed using the parallelizable sparse matrix linear algebra package MUMPS ${ }^{24,25}$ and the SPARSKIT2 library. ${ }^{26}$ These libraries exploit the high level of sparsity pattern offered by the finite-difference spatial differentiation, improving substantially on numerical efficiency while keeping accuracy; $\operatorname{see}^{27}$ for more details.

The $(\eta, \zeta)$ directions are discretized in a coupled manner using the stable high-order finite-differences numerical schemes of order $q$ (FD-q) developed in. ${ }^{28}$ These methods are used because of spectral-like accuracy is recovered, outperforming spectral collocation methods for stability analysis calculations in terms of accuracy and computational efficiency. ${ }^{27}$

Appropriate mappings between the finite-difference grids $(\xi \in[-1,1])$ and the computational domain are needed. Since the boundary layer problem requires clustering of points at the wall, the equation used to map the calculation domain grid $\eta \in[0,1]$ into the FD-q grid is

$$
\eta_{j}=l \frac{1-\xi_{j}}{1+s+\xi_{j}}, \quad s=2 l, \quad l=\frac{\eta_{h}}{1-2 \eta_{h}},
$$

$\eta_{h}$ being the domain location that splits in two halves the number of discretization points, fixed $\eta_{h}=0.2$. For the spanwise direction, the same transformation is used in order to cluster point near either the attachmentline, $\zeta=0$, or the top center-line, $\zeta=\pi / 2$.

\section{Eigenvalue computation}

The elliptic spatial BiGlobal problem, written as a GEVP in (16), is solved using the Arnoldi algorithm, ${ }^{29}$ delivering a number of eigenvalues in the vicinity of a specific estimated value, usually around the unstable/leaststable eigenvalue. Computational cost is substantially reduced when employing the Arnoldi algorithm instead of seeking the entire eigenspectrum as done by the classical QZ method. ${ }^{30}$ More details can be found in the literature. ${ }^{29,31}$

\section{Mean flow}

The geometry studied here corresponds to the HIFiRE-5 configuration. The HIFiRE program is a hypersonic flight test program executed by the Air Force Research Laboratory (AFRL) and the Australian Defense Science and Technology Organization (DSRO). ${ }^{32}$ The HIFiRE- 5 test payload consists of a blunt-nosed elliptic cone of 2:1 ellipticity, 0.86 meters in length. The nose tip cross-section in the minor axis describes a 2.5 $\mathrm{mm}$ radius circular arc, tangent to the cone ray describing the minor axis, and retains a 2:1 elliptical crosssection to the tip. Fight conditions were calculated for a Mach 7 flow at altitude of $33.0 \mathrm{~km}$ for Gosse et al. $^{33}$ The free-stream velocity is $2452.17 \mathrm{~m} / \mathrm{s}$ and the unit Reynolds number is $R e=1.89 \times 10^{6} / \mathrm{m}$. The surface temperature was defined using a prescribed temperature based on heat conduction analysis of an estimated 
trajectory for the vehicle. The mean flow solution has been calculated using the US3D non-equilibrium solver ${ }^{34}$ with shock fitting algorithm by Gosse et al. ${ }^{33}$ and has been used to extract the basic state analyzed here at a distance of 0.42 meters of the tip. Variables are nondimensionalized using the free-stream flow properties and the displacement thickness $\delta^{*}$ of the boundary layer profile at the top centerline as length scale, resulting $\delta^{*}=0.006 \mathrm{~m}$. At this location, the local displacement-thickness based Reynolds number value is $R e_{\delta^{*}}=11354$. Streamwise Mach number, $M a_{x}$, and base flow density are shown in Figure 1.

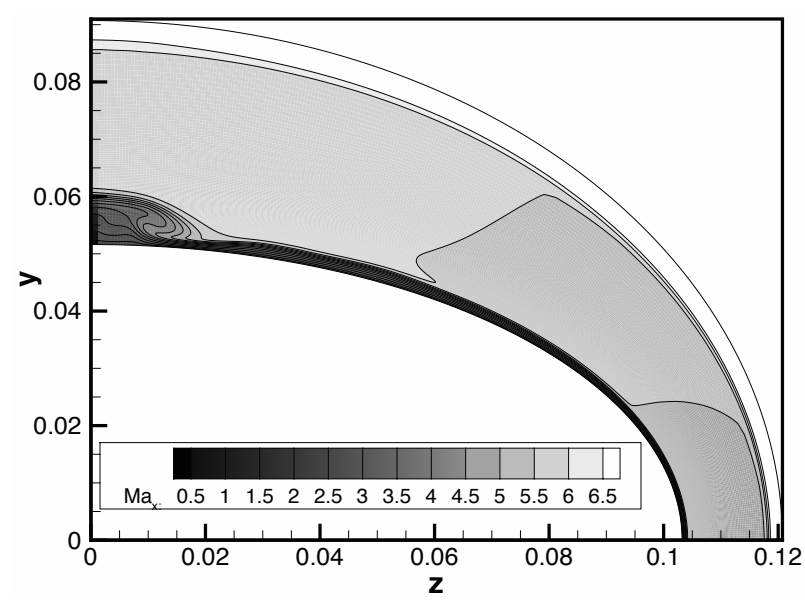

(a) $M a_{x}$

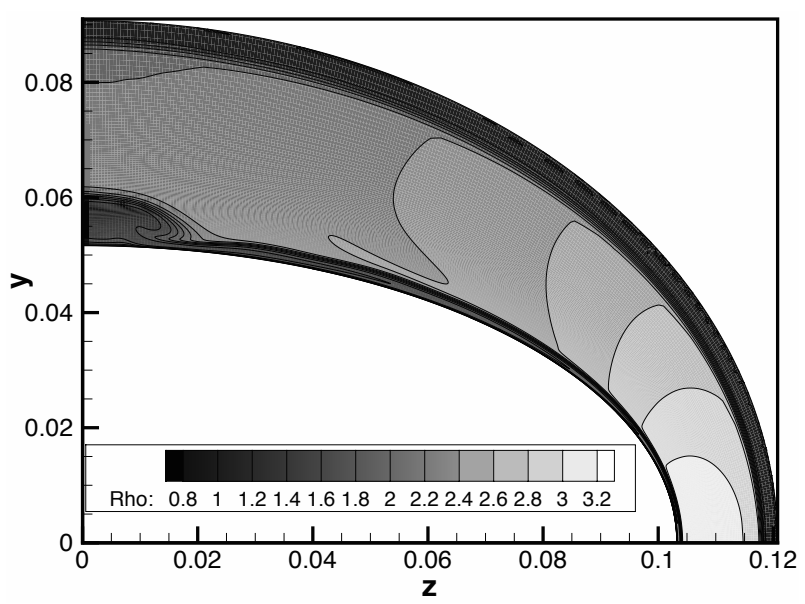

(b) $\bar{\rho}$

Figure 1. Streamwise Mach number and dimensionless density at $0.42 \mathrm{~m}$ from the cone vertex

\section{Results}

The previously mentioned mean flow is taken as base flow with $\mu_{\tau}=0^{33}$ for spatial BiGlobal stability analysis. The mean flow is interpolated into the grid constructed by Eq. (17). The analysis results show three different families of modes, the attachment-line modes, which structure is located near the major axis meridian and are well-understood in the incompressible ${ }^{35}$ and compressible ${ }^{36}$ regimes, the well-known crossflow modes ${ }^{37}$ and the center-line modes, which are accessible only to the present type of global eigenvalue problem solution.

\section{A. Attachment-line modes}

The eigenmodes peaking near the leading edge, ie. the major axis of the cone, are termed in the literature as attachment-line modes. The first studies on this flow started in 80's with the work of Hall et al. ${ }^{38}$ where the linear stability of the incompressible swept attachment-line boundary layer is studied, adopting the swept Hiemenz $^{39}$ basic flow and the Görtler-Hämmerlin ${ }^{40,41}$ similarity model for the perturbations. Instability in the incompressible orthogonal stagnation line flow was analyzed by Hall et al. ${ }^{38}$ as an (ODE-based) EVP, and by Theofilis ${ }^{42}$ as an initial value problem, both in a temporal context, while Theofilis ${ }^{43}$ solved the spatial EVP for this flow. Lin and Malik ${ }^{44}$ used a two-dimensional representation of the perturbations around the Hiemenz flow. Theofilis et al. ${ }^{35}$ performed temporal BiGlobal analysis of the incompressible orthogonal swept Hiemenz flow and proposed a polynomial model to describe the chordwise dependence of the amplitude functions, reducing the cost of global instability analysis without loss of physical information in the linear regime. The same methodology is used by Pérez et al. ${ }^{45}$ for the non-orthogonal case.

Study of compressibility effects on the leading edge boundary layer flow was introduced in a global analysis context by Theofilis et al. ${ }^{46}$ who solved a dense BiGlobal eigenvalue problem and presented an asymptotic theory along the lines of their earlier incompressible work. More recently, the compressible analyses of Mack \& Schmid ${ }^{47,36}$ in the swept cylinder body, identified families of modes of different (hydrodynamic or acoustic) physical origin. In the present elliptic cone flow, symmetric and antisymmetric attachment-lines modes, termed as $\mathrm{S}$ and A respectively in Table 1, are found to be highly unstable. Shape functions of these modes are showed in Figure 2. The parametric study of the attachment-line modes is currently underway. 
Table 1. Convergence of attachment-line spatially amplified global modes using FDq-6 with a linear mapping in the azimuthal direction. The two modes shown are identified with $\mathrm{A}$ and $\mathrm{S}$ in Figure 2.

\begin{tabular}{cccccc}
\hline & \multicolumn{3}{c}{$\mathrm{S}$} & \multicolumn{2}{c}{$\mathrm{A}$} \\
$\mathrm{Nz}$ & $\mathrm{Ny}$ & $\alpha_{r}$ & $-\alpha_{i}$ & $\alpha_{r}$ & $-\alpha_{i}$ \\
\hline 301 & 141 & 2.0760 & 0.39636 & 2.03474 & 0.42014 \\
321 & 141 & 2.0634 & 0.39237 & 2.28225 & 0.43113 \\
\hline
\end{tabular}

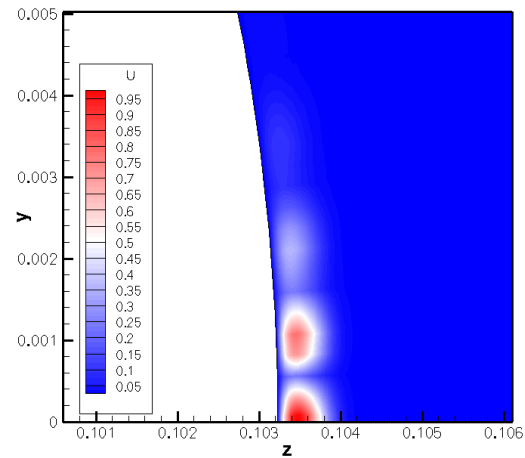

(a) $\mathrm{S},\|\hat{u}\|$

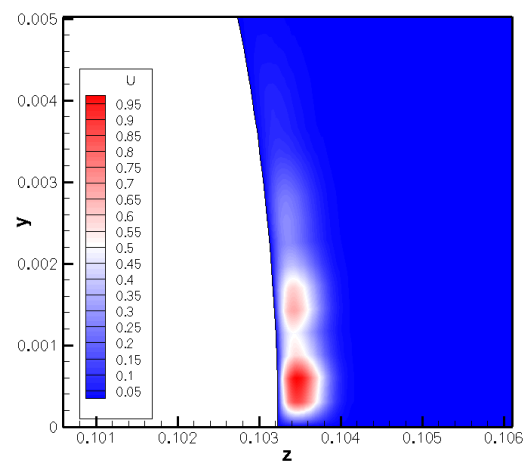

(d) $\mathrm{A},\|\hat{u}\|$

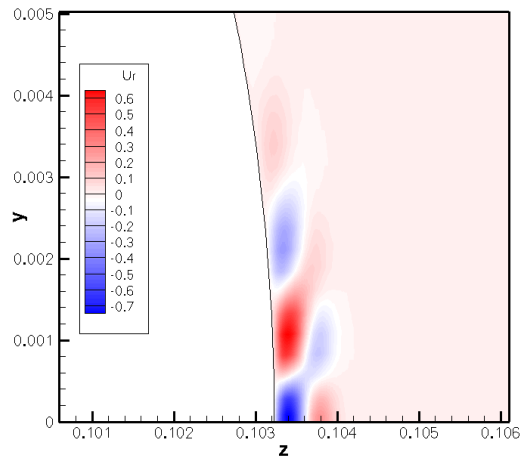

(b) S, $\hat{u}_{r}$

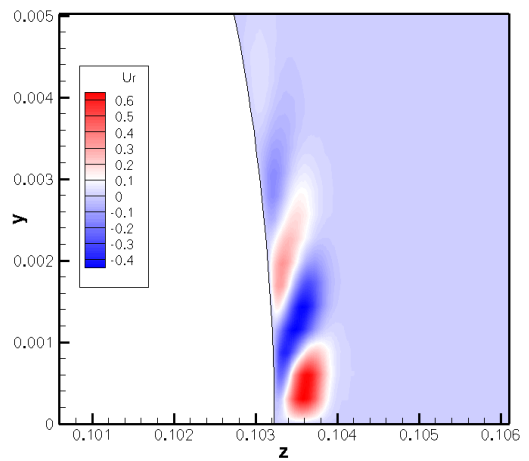

(e) A, $\hat{u}_{r}$

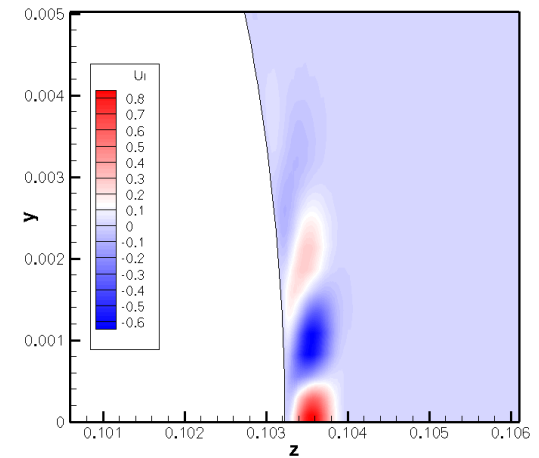

(c) $\mathrm{S}, \hat{u}_{i}$

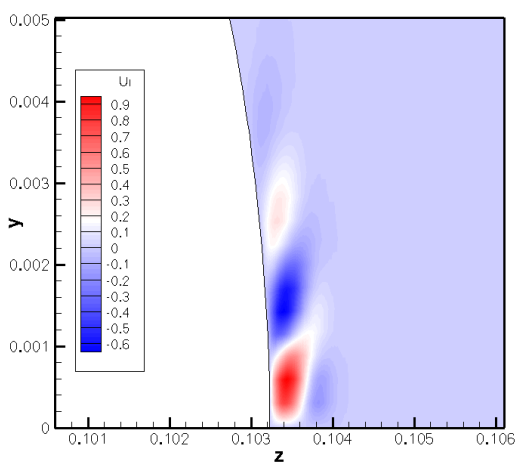

(f) $\mathrm{A}, \hat{u}_{r}$

Figure 2. Streamwise velocity shape functions of attachment-line eigenmodes for $\omega=1.0$. Same abbreviations as in Table 1. 


\section{B. Cross-flow modes}

In three-dimensional boundary layers, the cross-flow instabilities can become the dominant path to boundary layer transition; see. ${ }^{20,48,49}$ Any three-dimensionality inevitably produces spanwise pressure gradients. These gradients induce cross-flow, where the flow direction of the interior of the boundary layer is no longer co-planar with the edge velocity vector. The low-momentum boundary layer fluid near the surface is deflected more than the fluid near the edge of the boundary layer. In these conditions cross-flow vortices may be established, which may dominate the transition process. The recent work of Borg et al. ${ }^{50}$ investigated experimentally both the stationary cross-flow instability and traveling disturbances as arising on the same elliptic cone geometry as that presently studied. These authors observed stationary cross-flow vortices and co-located traveling disturbances. The cross-flow velocity, defined as

$$
V_{C F}=\frac{\bar{u} \zeta_{x}+\bar{v} \zeta_{y}+\bar{w} \zeta_{z}}{\sqrt{\zeta_{x}^{2}+\zeta_{y}^{2}+\zeta_{z}^{2}}},
$$

is plotted in Figure 3 at the studied cross-section of the cone. The existence of this velocity leads to the appearance of cross-flow instabilities. Several amplified cross-flow modes were observed in the spatial BiGlobal eigenspectra recoverd in the present analysis. The wavenumbers and growth rates of two of these modes, CF1 and CF2, are shown in Table 2. Shape functions of these modes are plotted in Figure 4; they are shown to peak at locations where the cross-flow velocity is maximized. The parametric study of the cross-flow modes is currently also underway.

Table 2. Convergence of cross-flow spatially amplified global modes using FDq-6. The two modes shown are identified with CF1 and CF2 in Figure 4.

\begin{tabular}{cccccc}
\hline & \multicolumn{2}{c}{ CF1 } & \multicolumn{2}{c}{ CF2 } \\
$\mathrm{Nz}$ & $\mathrm{Ny}$ & $\alpha_{r}$ & $-\alpha_{i}$ & $\alpha_{r}$ & $-\alpha_{i}$ \\
\hline 301 & 141 & 0.8367351 & 0.1020891 & 0.4726402 & 0.0868370 \\
321 & 141 & 0.8360357 & 0.1023844 & 0.4747186 & 0.0914797 \\
\hline
\end{tabular}

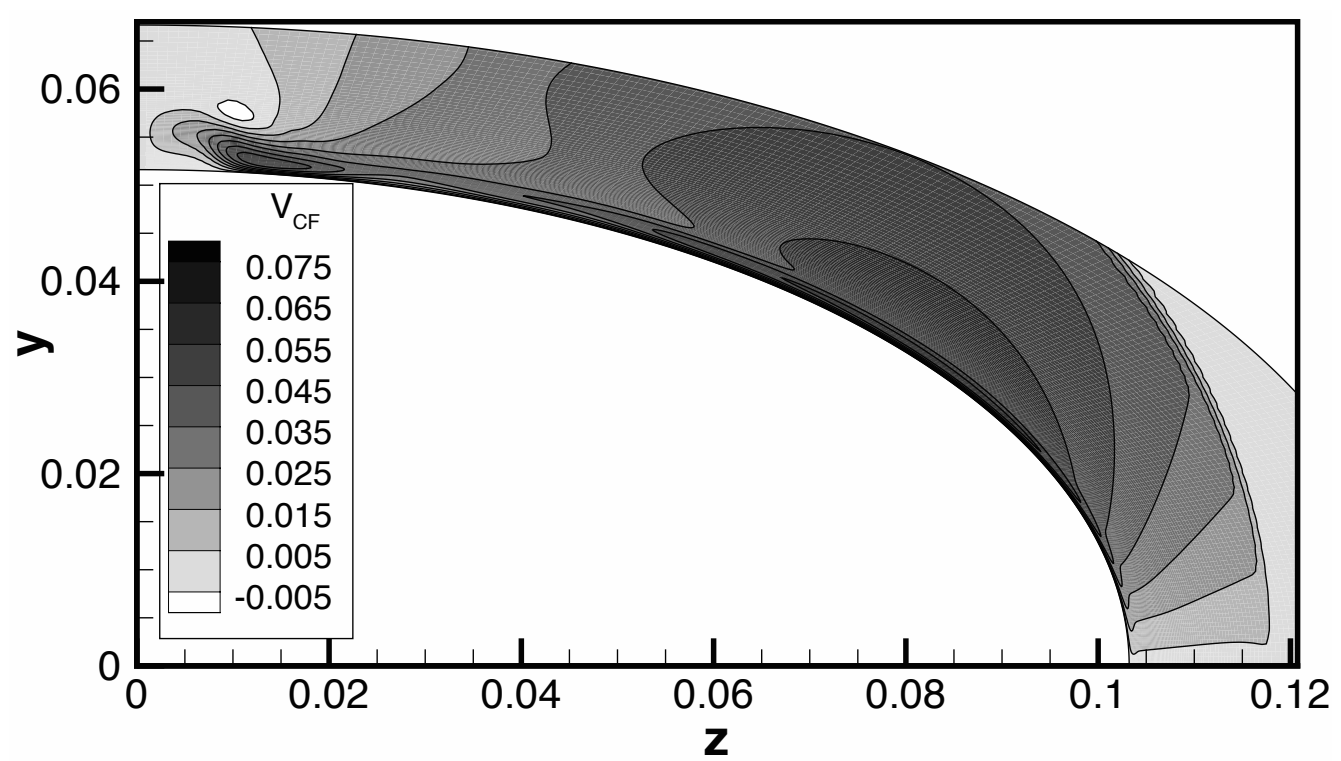

Figure 3. Dimensionless base flow cross-flow velocity near the wall. 


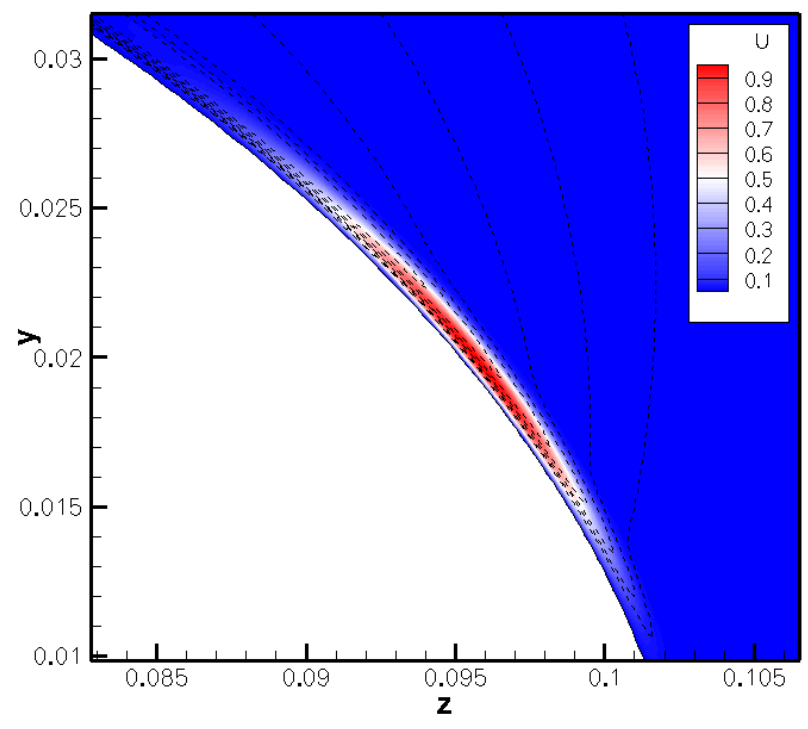

(a) $\mathrm{CF} 1,\|\hat{u}\|$

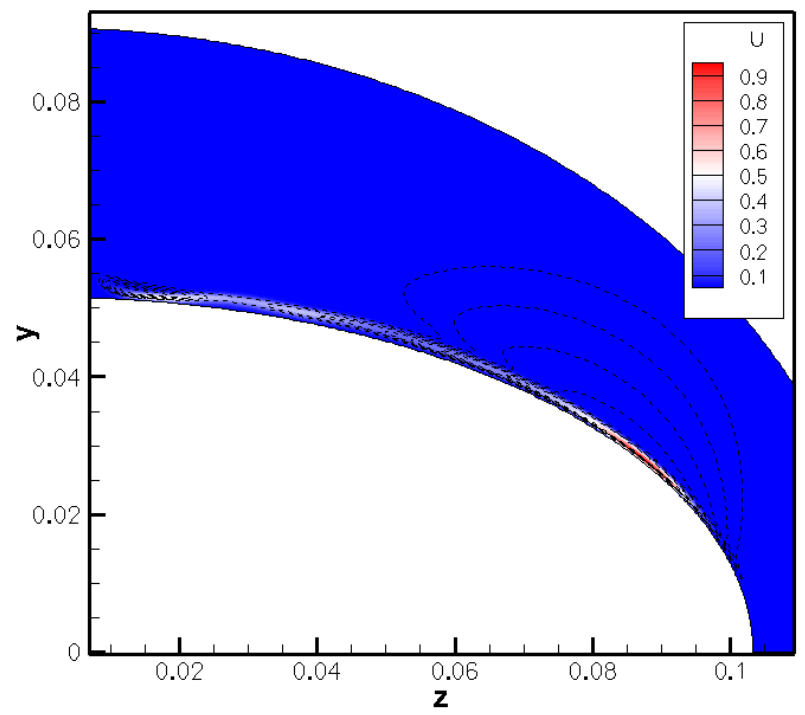

(c) $\mathrm{CF} 2,\|\hat{u}\|$

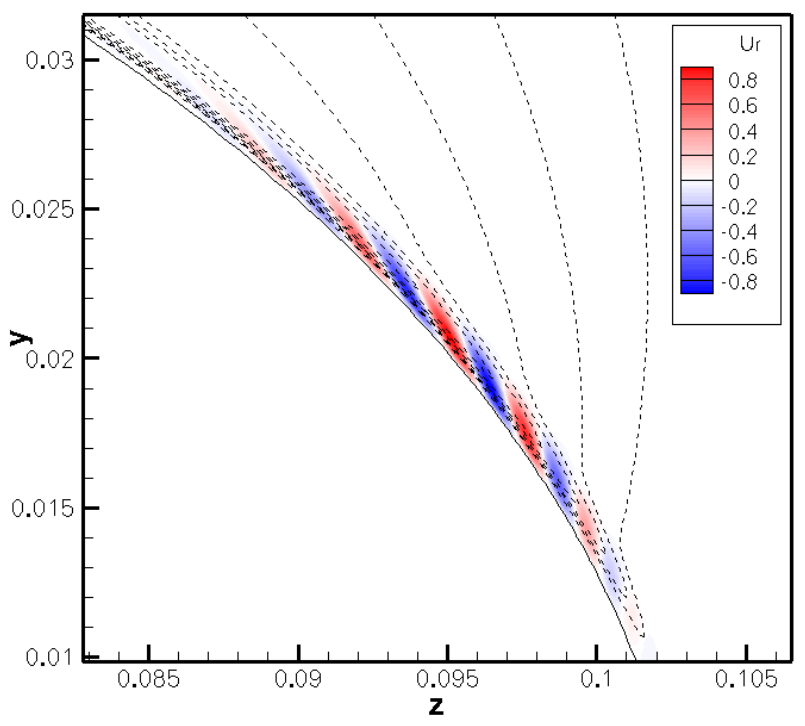

(b) CF1, $\hat{u}_{r}$

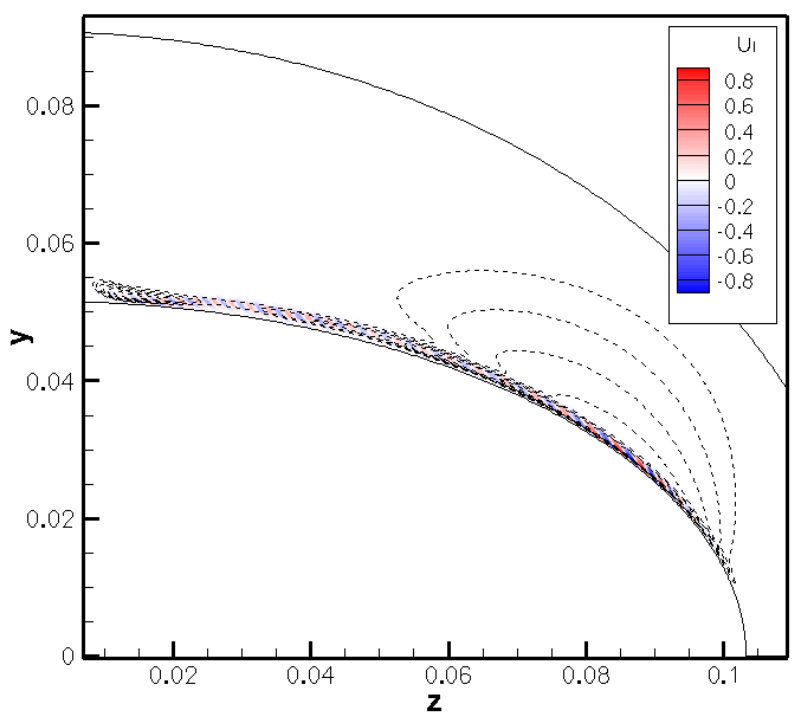

(d) CF2, $\hat{u}_{r}$

Figure 4. Streamwise velocity shape functions of cross-flow eigenmodes for $\omega=1.0$. Same abbreviations as in Table 2. Dashed lines refers to iso-lines of dimensionless base flow cross-flow velocity $\bar{v}_{C F}=0.045:(0.005): 0.075$. Imaginary parts of eigenmodes are identical to real parts but with different phase angle. 


\section{Center-line modes}

The bulk of the efforts in the present analysis was devoted to documenting the center-line modes, for a wide range of frequencies. In line with the analogous finding in the swept attachment line ${ }^{44}$ a sequence of amplified modes was discovered, when employing symmetric and anti-symmetric boundary conditions to the solution of the spatial BiGlobal eigenvalue problem. Figure 5 shows the wavenumber and growth rate of the four leading center-line modes in the studied branch of frequencies. A symmetric mode, S1, is found to be the most amplified one, peaking at frequency around $F^{*}=130 \mathrm{kHz}$. As it is observed in Figure $5(\mathrm{~b})$, this mode is followed by an antisymmetric mode, A1. Table 3 shows the convergence history of the symmetric modes. The streamwise velocity shape functions of these center-line modes are plotted in Figure 6. Similar structure to the S1 mode was found by Choudhari et al. ${ }^{16}$ who analyzed through a two-dimensional stability theory the structures near the top center-line of the flow, without accounting for surface curvature effects. Work is underway to complete the characterization of the center-line modes.

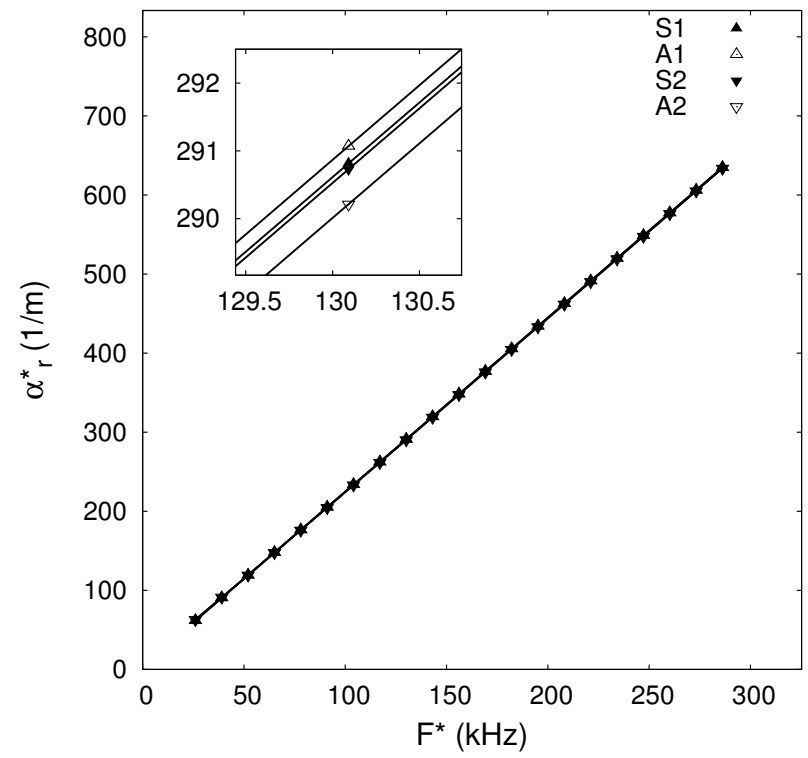

(a)

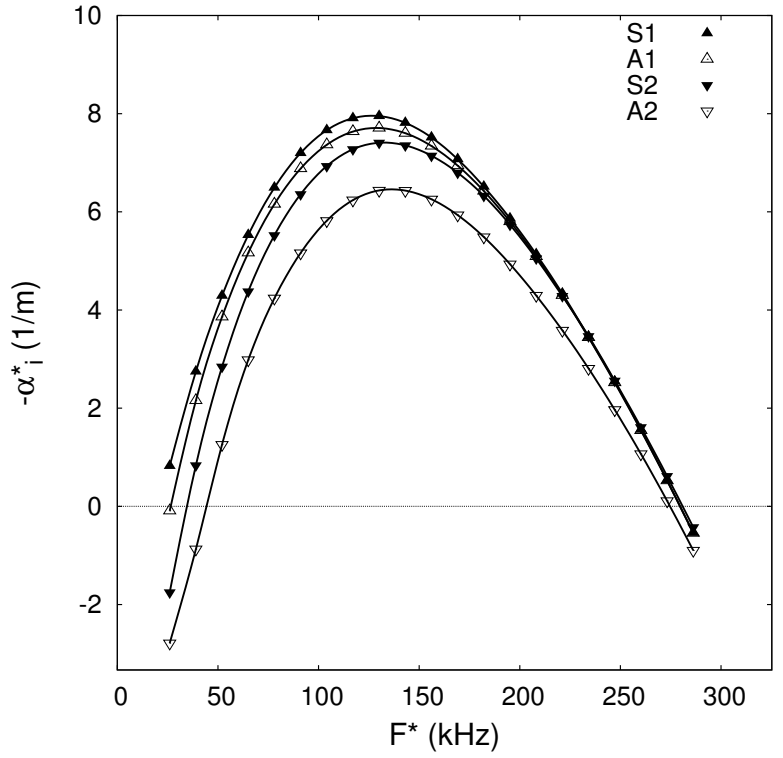

(b)

Figure 5. Wavenumber and growth rate of most amplified center-line modes for different frequencies. Note that $\mathbf{A}$ refers to antisymmetric and $\mathbf{S}$ to symmetric boundary conditions at $\zeta=0$ and $\zeta=\pi / 2$.

Table 3. Convergence of center-line spatially amplified global modes using FDq-6, together with the streching mapping of Eq. 18 for the azimuthal coordinate, setting $\zeta_{h}=3 \pi / 8$. The two modes shown are identified with S1 and S2 in Figure 5. Similar convergence has been observed for the A1 and A2 modes.

\begin{tabular}{cccccc}
\hline & \multicolumn{3}{c}{$\mathrm{S} 1$} & \multicolumn{2}{c}{$\mathrm{S} 2$} \\
$\mathrm{Nz}$ & $\mathrm{Ny}$ & $\alpha_{r}$ & $-\alpha_{i}$ & $\alpha_{r}$ & $-\alpha_{i}$ \\
\hline 141 & 141 & 0.8878996 & 0.0309837 & 0.8854285 & 0.0179014 \\
161 & 141 & 0.8878997 & 0.0309838 & 0.8854284 & 0.0179011 \\
151 & 151 & 0.8879054 & 0.0309825 & 0.8854505 & 0.0179220 \\
\hline
\end{tabular}

\section{Conclusions}

We present the first application of spatial BiGlobal linear theory to the analysis of instability mechanisms on the elliptic cone in flow over a 2:1 elliptic cone at $M a=7$. The main finding is the identification of three different families of spatially amplified linear global modes. The attachment-line and the cross-flow modes are 


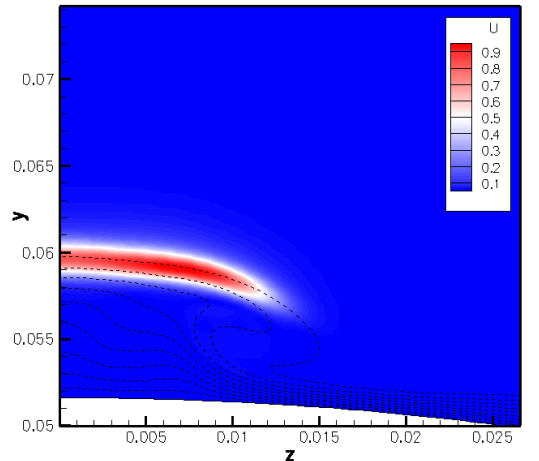

(a) $\mathrm{S} 1,\|\hat{u}\|$

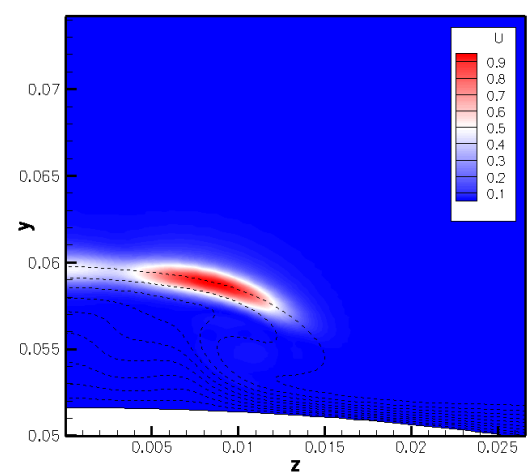

(d) S2, $\|\hat{u}\|$

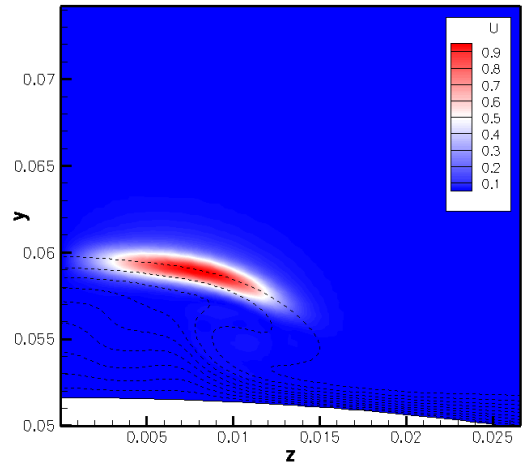

(g) A1, $\|\hat{u}\|$

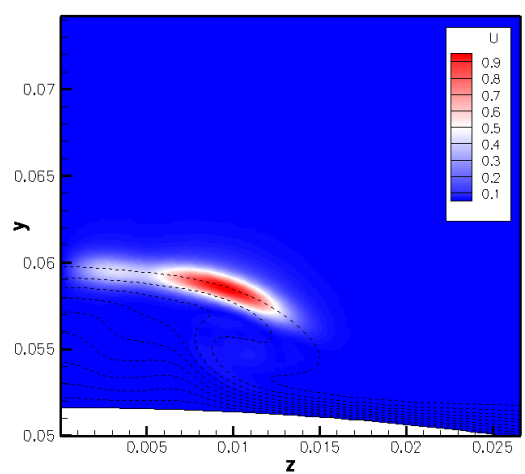

(j) A2, $\|\hat{u}\|$

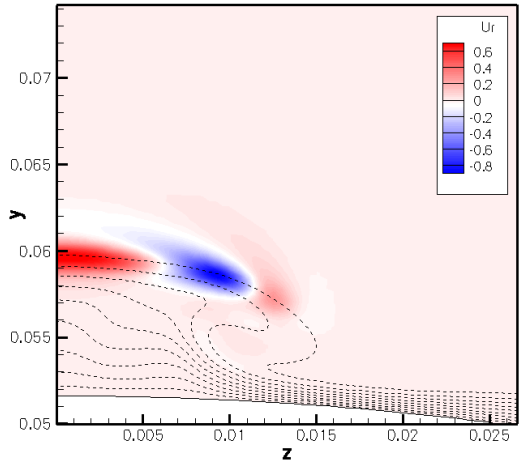

(b) $\mathrm{S} 1, \hat{u}_{r}$

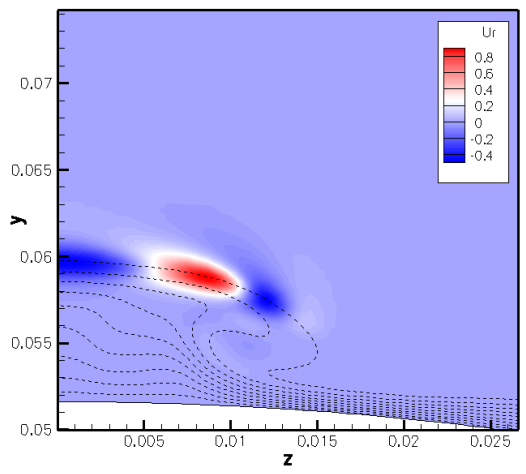

(e) $\mathrm{S} 2, \hat{u}_{r}$

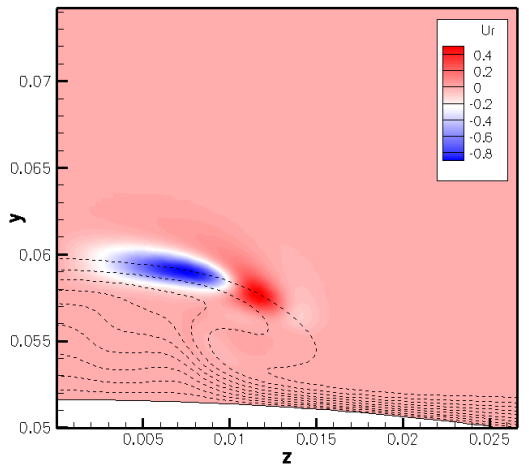

(h) A1, $\hat{u}_{r}$

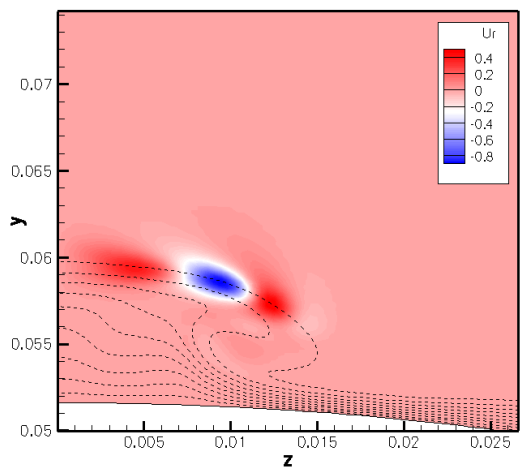

(k) A2, $\hat{u}_{r}$

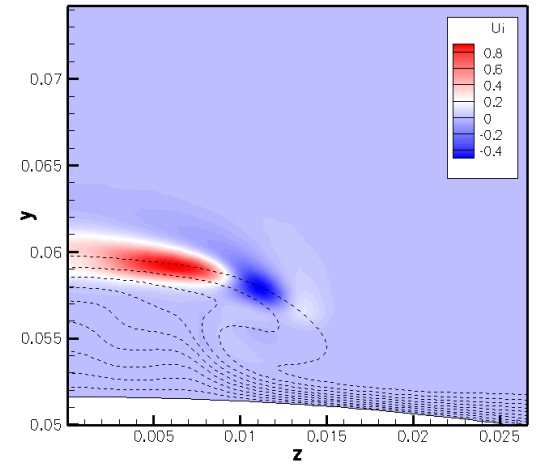

(c) $\mathrm{S} 1, \hat{u}_{i}$

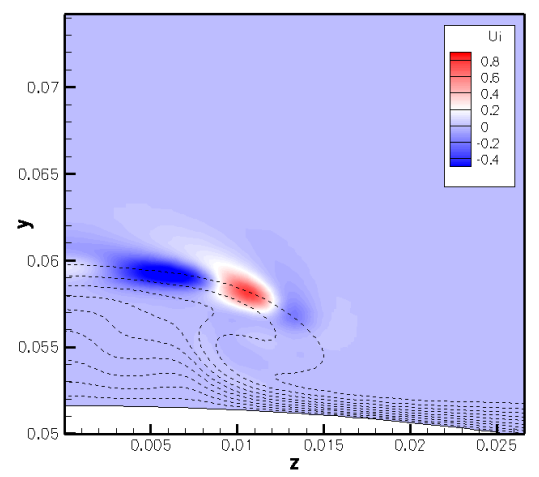

(f) $\mathrm{S} 2, \hat{u}_{i}$

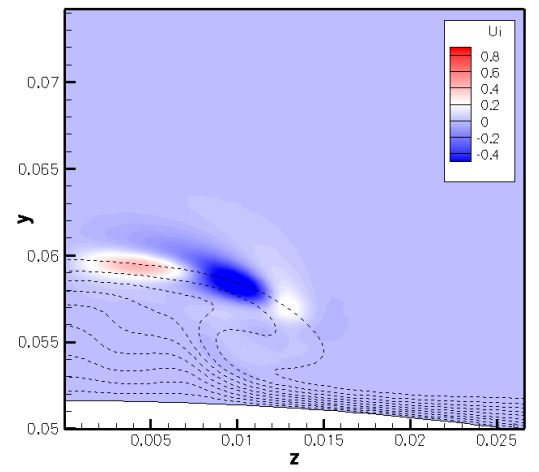

(i) $\mathrm{A} 1, \hat{u}_{i}$

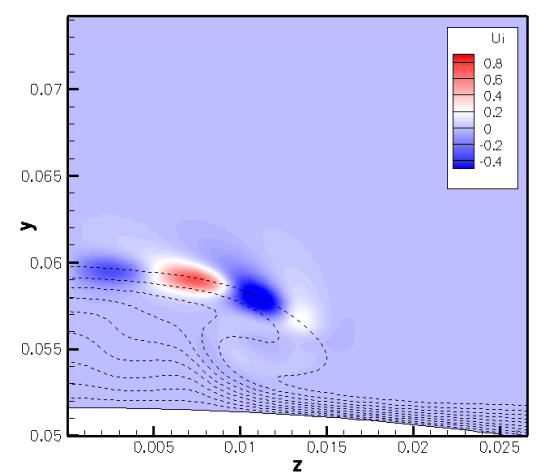

(l) $\mathrm{A} 2, \hat{u}_{i}$

Figure 6. Streamwise velocity shape functions of center-line eigenmodes for $\omega=2.0\left(F^{*}=130 \mathrm{kHz}\right)$. Same abbreviations as in Figure 5. Dashed lines refers to iso-lines of dimensionless base flow streamwise velocity $\bar{u}=0.1:(0.1): 0.9$ 
known from earlier local and non-local analyses, ${ }^{16}$ while a new linear global eigenmode is identified, termed the center-line mode, which is accessible only to the present type of global eigenvalue problem solutions. In a manner reminiscent of the analogous sequence of attachment-line modes in leading-edge boundary layer flow, we discover that a sequence of symmetric and anti-symmetric center-line modes exist and, for the studied flow conditions, height of $33 \mathrm{~km}$, unit Reynolds number $1.89 \times 10^{6} / \mathrm{m}$ and axial section of the cone at a distance of $0.42 \mathrm{~m}$ from the tip, are maximally amplified around $F^{*}=130 \mathrm{kHz}$. Results show that the attachment-line and cross-flow modes have higher growth rates than the center-line modes at these conditions. A mapping of the parameter space with respect to critical conditions is underway.

\section{Acknowledgments}

The authors thank Dr. Ryan Gosse and Dr. Roger Kimmel for their collaboration supplying the main flow. Effort sponsored by the Air Force Office of Scientific Research, Air Force Material Command, USAF, under grant number FA8655-12-1-2004. The U.S Government is authorized to reproduce and distribute reprints for Governmental purpose notwithstanding any copyright notation thereon.

\section{References}

${ }^{1}$ Schmisseur, J., Schneider, S., and Collicott, S., "Receptivity of the Mach-4 boundary-layer on an elliptic cone to lasergenerated localized free stream perturbations," AIAA Paper 98-0532, 1998.

${ }^{2}$ Schmisseur, J., Schneider, S., and Collicott, S., "Response of the Mach-4 boundary layer on an elliptic cone to lasergenerated free stream perturbations," AIAA Paper 99-0410, 1999.

${ }^{3}$ Poggie, J. and Kimmel, R., "Traveling instabilities in elliptic cone boundary-layer transition at Ma=8," AIAA Paper 98-0435, 1998.

${ }^{4}$ Huntley, M. and Smits, A., "Transition studies on an elliptic cone in Mach 8 flow using filtered Rayleigh scattering," European Journal of Mechanics B - Fluids, Vol. 19, No. 5, 2000, pp. 695-706.

${ }^{5}$ Bartkowicz, M., Subbareddy, P., and Candler, G., "Simulation of boundary layer transition of elliptic cones in hypersonic flow," AIAA Paper 2010-1064, 2010.

${ }^{6}$ Mack, L. M., "Boundary layer linear stability theory," AGARD-R-709 Special course on stability and transition of laminar flow, 1984, pp. 3.1-3.81.

${ }^{7}$ Lyttle, I. and Reed, H., "Use of transition correlations for three-dimensional boundary layers within hypersonic flows," AIAA Paper 95-2293, 1995.

${ }^{8}$ Reed, H. and Haynes, T., "Transition correlations in three-dimensional boundary layers," AIAA J., Vol. 32, 1994, pp. 923-929.

${ }^{9}$ Kimmel, R., Klein, M., and Schwoerke, S., "Three-dimensional hypersonic laminar boundary-layer computations for transition experiment design," J. Spacecraft Rockets, Vol. 34, No. 4, 1997, pp. 409-415.

${ }^{10}$ Lawrence, S., Tannehill, J., and Chaussee, D., "An upwind algorithm for the parabolized Navier-Stokes equations," AIAA Paper, Vol. 86, 1986, pp. 1117.

${ }^{11}$ Stalnaker, J., Nicholson, L., Hanline, D., and McGraw, E., "Improvements to the AFWAL parabolized Navier-Stokes code formulation," U.s. airforce wright aeronautical labs., tr afwal-tr-86-3076, Wright-Patterson AFB, OH, 1986.

${ }^{12}$ Malik, M., "e Malik: a new spatial stability analysis program for transition prediction using the $\mathrm{e}^{N}$ method," Rept. htc-8902, High Technology Corp., Hampton, VA, 1989.

${ }^{13}$ Poggie, J., Kimmel, R., and Schwoerke, S., "Traveling instability waves in a Mach 8 flow over an elliptic cone," AIAA J., Vol. 38, No. 2, 2000, pp. 251-258.

${ }^{14}$ Gosse, R. and Kimmel, R., "CFD study of three-dimensional hypersonic laminar boundary layer transition on a Mach 8 elliptic cone," AIAA Paper 2009-4053, 2009.

${ }^{15}$ Kimmel, R., Adamczak, D., Berger, K., and Choudhari, M., "HIFiRE-5 flight vehicle design," AIAA Paper 2010-4985, 2010.

${ }^{16}$ Choudhari, M., Chang, C., Jentink, T., Li, F., Berger, K., Candler, G., and Kimmel, R., "Transition analysis for the HIFiRE-5 vehicle," AIAA Paper 2009-4056, 2009.

${ }^{17}$ Li, F., Choudhari, M., Chang, C., White, J., Kimmel, R., Adamczak, D., Borg, M., Stanfield, S., and Smith, M., "Stability analysis for HIFiRE experiments," AIAA Paper 2012-2961, 2012

${ }^{18}$ Theofilis, V., "Global linear instability," Annu. Rev. Fluid Mech., Vol. 43, 2011, pp. 319-352.

${ }^{19}$ De Tullio, N., Paredes, P., Sandham, N., and Theofilis, V., "Roughness-induced instability and breakdown to turbulence in a supersonic boundary-layer," J. Fluid Mech., Vol. (submitted), 2013.

${ }^{20}$ Reed, H., Saric, W., and Arnal, D., "Linear stability theory applied to boundary layers," Ann. Rev. Fluid Mech., Vol. 28, 1996, pp. 389-428. 171.

${ }^{21}$ Theofilis, V., "Spatial stability of incompressible attachment line flow," Theor. Comput. Fluid Dyn., Vol. 7, 1995, pp. 159-

${ }^{22}$ Bridges, T. and Morris, P., "Differential eigenvalue problems in which the parameter appears nonlinearly," J. Comp. Phys., Vol. 55, 1984, pp. 437-460. 

2013.

${ }^{23}$ Paredes, P., The PSE-3D instability methodology for complex flows, Ph.D. thesis, Universidad Politécnica de Madrid,

${ }^{24}$ Amestoy, P., Duff, I., Koster, J., and L'Excellent, "A fully asynchronous multifrontal solver using distributed dynamic scheduling," SIAM Journal of Matrix Analysis and Applications., Vol. 1, 2001, pp. 15-41.

${ }^{25}$ Amestoy, P., Guermouche, A., L'Excellent, J.-Y., and Pralet, S., "Hybrid scheduling for the parallel solution of linear systems," Parallel Computing, Vol. 2, 2006, pp. 136-156.

${ }^{26}$ Saad, Y., "SPARSKIT: a basic tool kit for sparse matrix computations, Version 2." 1994.

${ }^{27}$ Paredes, P., Hermanns, M., Le Clainche, S., and Theofilis, V., "Order $10^{4}$ speedup in global linear instability analysis using matrix formation," Comput. Meth. Appl. Mech. Eng., Vol. 253, 2013, pp. 287-304.

${ }^{28}$ Hermanns, M. and Hernández, J. A., "Stable high-order finite-difference methods based on non-uniform grid point distributions," Int. J. Numer. Meth. Fluids, Vol. 56, 2008, pp. 233-255.

${ }^{29}$ Saad, Y., "Variations of Arnoldi's method for computing eigenelements of large unsymmetric matrices," Linear Algebra Applications, Vol. 34, 1980, pp. 269-295.

${ }^{30}$ Wilkinson, J. H., The algebraic eigenvalue problem, Clarendon, 1965.

${ }^{31}$ Theofilis, V., "Advances in global linear instability of nonparallel and three-dimensional flows," Prog. Aero. Sciences, Vol. 39 (4), 2003, pp. 249-315.

${ }^{32}$ Dolvin, D., "Hypersonic Iinternational Flight Research and Eexperimentation (HIFiRE) fundamental science and technology development strategy," AIAA Paper 2008-2581, 2008.

${ }^{33}$ Gosse, R., Kimmel, R., and Johnson, H., "CFD study of the HIFiRE-5 flight experiment," AIAA Paper 2010-4854, 2010.

${ }^{34}$ Nompelis, I., Drayna, T., and Candler, G., "A parallel unstructured implicit solver for hypersonic reacting flow simulation," AIAA Paper 2005-4867, 2005.

${ }^{35}$ Theofilis, V., Fedorov, A., Obrist, D., and Dallmann, U. C., "The extended Görtler-Hämmerlin model for linear instability of three-dimensional incompressible swept attachment-line boundary layer flow," J. Fluid Mech., Vol. 487, 2003 , pp. $271-313$.

${ }^{36}$ Mack, C. J. and Schmid, P. J., "Global stability of swept flow around a parabolic body: features of the global spectrum," J. Fluid Mech., Vol. 669, 2011, pp. 375-396.

${ }^{37} \mathrm{Li}, \mathrm{F}$. and Choudhari, M., "Spatially developing secondary instabilities in compressible swept airfoil boundary layers," Theor. Comp. Fluid Dyn., Vol. 25, 2011, pp. 65-84.

${ }^{38}$ Hall, P., Malik, M. R., and Poll, D. I. A., "On the stability of an infinitive swept attachment line boundary layer," Proc. R. Soc. Lond. A, Vol. 395, 1984, pp. 229-245.

${ }^{39}$ Hiemenz, K., "Die Grenzschicht an einem in den gleichförmigen Flüssigkeitsstrom eingetauchten geraden Kreiszylinder," Dingl. Polytechn. J., Vol. 326, 1911, pp. 321-324, Thesis, Göttingen.

${ }^{40}$ Görtler, H., "Dreidimensionale Instabilität der ebenen Staupunktströmung gegenüber wirbelartigen Störungen," 50 Jahre Grenzschichtforschung, edited by H. Görtler and W. Tollmien, Vieweg und Sohn, 1955, pp. 304-314.

${ }^{41}$ Hämmerlin, G., "Zur instabilitätstheorie der ebenen Staupunktströmung," 50 Jahre Grenzschichtforschung, edited by H. Görtler and W. Tollmien, Vieweg und Sohn, 1955, pp. 315-327.

${ }^{42}$ Theofilis, V., "Numerical experiments on the stability of leading-edge boundary-layer flow: a 2-dimensional linear study," Int. J. Numer. Meth. Fluids, Vol. 16, No. 2, 1993, pp. 153-170.

${ }^{43}$ Theofilis, V., "Spatial stability of incompressible attachment-line flow," Theor. Comp. Fluid Dyn., Vol. 7, No. 3, 1995, pp. 159-171.

${ }^{44}$ Lin, R. S. and Malik, M. R., "On the stability of attachment-line boundary layers. Part 1. The incompressible swept Hiemenz flow," J. Fluid Mech., Vol. 311, 1996, pp. 239-255.

${ }^{45}$ Pérez, J. M., Rodríguez, D., and Theofilis, V., "Linear global instability of non-orthogonal incompressible swept attachment-line boundary-layer flow," J. Fluid Mech., Vol. DOI: http://dx.doi.org/10.1017/jfm.2012.354, 2012, pp. (to appear).

${ }^{46}$ Theofilis, V., Fedorov, A. V., and Collis, S. S., "Leading-edge boundary layer flow - Prandtl's vision, current developments and future perspectives," IUTAM Symposium on One Hundred Years of Boundary Layer Research, Vol. 129, 2004.

${ }^{47}$ Mack, C. J. and Schmid, P. J., "A preconditioned Krylov technique for global hydrodynamic stability analysis of lardescale compressible flows," J. Comp. Phys., Vol. 229, No. 3, 2010, pp. 541-560.

${ }^{48}$ Reed, H. L. and Saric, W. S., "Stability of three-dimensional boundary layers," Annu. Rev. Fluid Mech., Vol. 21, 1989, pp. 235-284.

${ }^{49}$ Reed, H. L., Saric, W. S., and Arnal, D., "Linear stability theory applied to boundary layers," Annu. Rev. Fluid Mech., Vol. 28, 1996, pp. 389-428.

${ }^{50}$ Borg, M., Kimmel, R., and Stanfield, S., "Crossflow instability for HIFiRE-5 in a quiet hypersonic wind tunnel," AIAA Paper 2012-2821, 2012. 